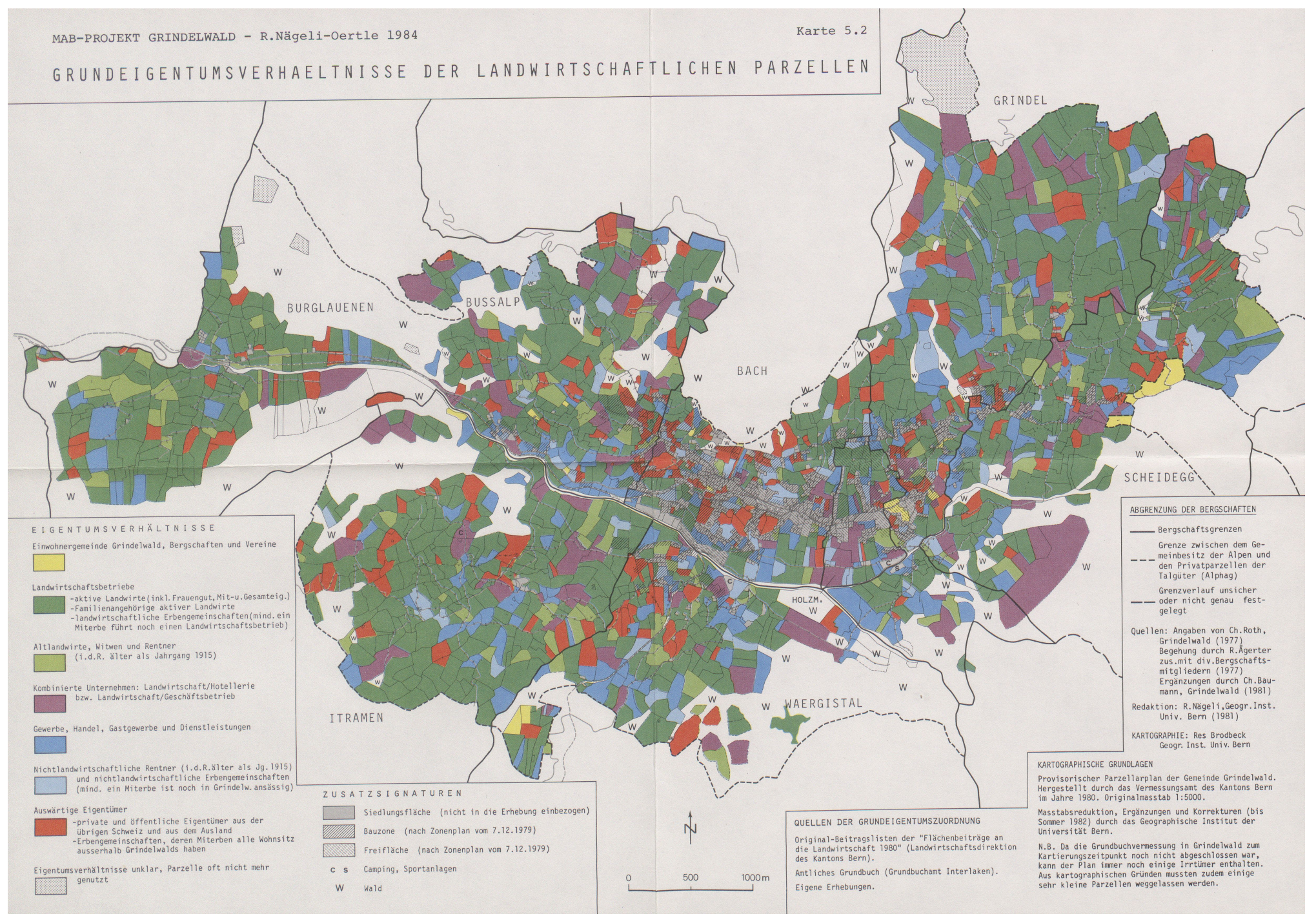




\section{Veränderungen der landwirtschaftlichen Betriebs- und Grundeigentumsstruktur als Abbild des sozialökonomischen Wandels im Berggebiet: das Beispiel Grindelwald}

(mit Kartenbeilage)

Im Rahmen des nun mehrheitlich abgeschlossenen Forschungsprojektes MAB [Man and Biosphere] Grindelwald $^{1}$ ist auch die dortige Berglandwirtschaft einer vertieften Struktur- und Entwicklungsanalyse unterzogen worden (vgl. NAEGELIOERTLE 1986a und 1986b).

$A n l a ß$ und Ziel des folgenden Beitrages ist es, eine im Rahmen dieses Projektes erstellte flächendekkende Kartierung des landwirtschaftlichen Grundeigentums in Grindelwald vorzustellen und in einigen Aspekten zu erläutern. ${ }^{2}$ Die dazugehörende Karte im Maßstab 1:25000 ist diesem Heft als separate Falzkarte unter dem Titel «MAB-Projekt Grindelwald - Grundeigentumsverhältnisse der landwirtschaftlichen Parzellen» beigelegt .

Damit diese statische Situationsaufnahme der Grundeigentumsstruktur als Zeitschnitt einer an sich dynamischen Entwicklung und Veränderung der Landwirtschaft im touristisch orientierten Berggebiet interpretiert werden kann, sind vorab einige Hinweise zum betrieblichen und räumlichen Strukturwandel der Grindelwalder Landwirtschaft erforderlich (vgl. zur räumlichen Orientierung die Abb. 1).

\section{Veränderungen der Betriebsstruktur}

Ähnlich wie in vielen anderen touristisch entwickelten Gemeinden im Berner Oberland (vgl. STALDER 1988 i. E.) bzw. im weiteren schweizerischen Berggebiet ist seit etwa 20 Jahren auch in Grindelwald die Berglandwirtschaft einem beträchtlichen Strukturwandel unterworfen.

Dieser Veränderungsprozeß läßt sich für Grindelwald unter anderem durch die folgenden Merkmale charakterisieren:

- eine beträchtliche Abnahme der Anzahl Betriebe (von über $320 \mathrm{im}$ Jahr 1965 auf rund 246 Betriebe im Jahr 1985);

- parallel dazu jedoch ein markanter Anstieg der durchschnittlichen Betriebsgröße (im statistischen Durchschnitt im gleichen Zeitraum von 3,6 ha auf 6,8 ha; eine Analyse der Betriebsveränderungen nach Flächengrößenklassen deckt jedoch auf, daß v. a. die Betriebe mit einer Fläche von $<5$ ha abgenommen haben, während sich die Gruppe mit 5-10 ha relativ stabil verhalten hat, und die Betriebe mit $>10$ ha deutlich zugenommen haben;

- eine drastische Reduktion der landwirtschaftlichen Arbeitskräfte (von über 500 ständigen Arbeitskräften um 1960 auf rund 130 im Jahr 1985);

- eine rasch fortschreitende Außenmechanisierung (wobei v. a. Transporter, Ladewagen, Kreiselheuer, Mistzetter und neuerdings die vierradgetriebenen Hangtraktoren im Vordergrund stehen);

- gefolgt von einer eher zögernden Innenmechanisierung (Melkanlagen, Silos, Heubelüftungen) und Zentralisierung der verstreuten Betriebsgebäude (um 1981 wiesen erst 18 Betriebe eine neue Zentralscheune auf);

- sowie schließlich eine immer dominantere Bedeutung des nichtlandwirtschaftlichen Erwerbs (um 1980 übten nach einer eigenen Vollerhebung 72\% der Betriebsleiter im Erwerbsalter eine teil- oder ganzzeitliche nichtlandwirtschaftliche Erwerbstätigkeit aus, $20 \%$ standen im AHV-Alter, nur die restlichen $8 \%$ lebten ausschließlich von der Landwirtschaft).

Eine detailliertere Analyse der einzelbetrieblichen Entwicklungsdynamik macht deutlich, daß trotz des massiven Rückganges der Betriebszahl und der Arbeitskräfte im Falle von Grindelwald bisher nicht von einer besorgniserregenden Rückbildung der Landwirtschaft gesprochen werden muß. Der Viehbestand hat sich über die ganze Periode hinweg mehr oder weniger auf der gleichen Höhe gehalten. Hinsichtlich der Flächennutzung ist jedoch ein gewisser Rückzug aus den Grenzertragsstandorten der Gemeindeperipherie festzustellen.

Eine Veränderung, welche sich seit den letzten 10 bis 15 Jahren jedoch deutlich abzeichnet, und welche langfristig gesehen negative Folgen für das gesamte Landnutzungsgefüge haben könnte, ist eine

Ruedi Nägeli-Oertle, Dr., Geographisches Institut der Universität Bern, Hallerstraße 12, 3012 Bern 


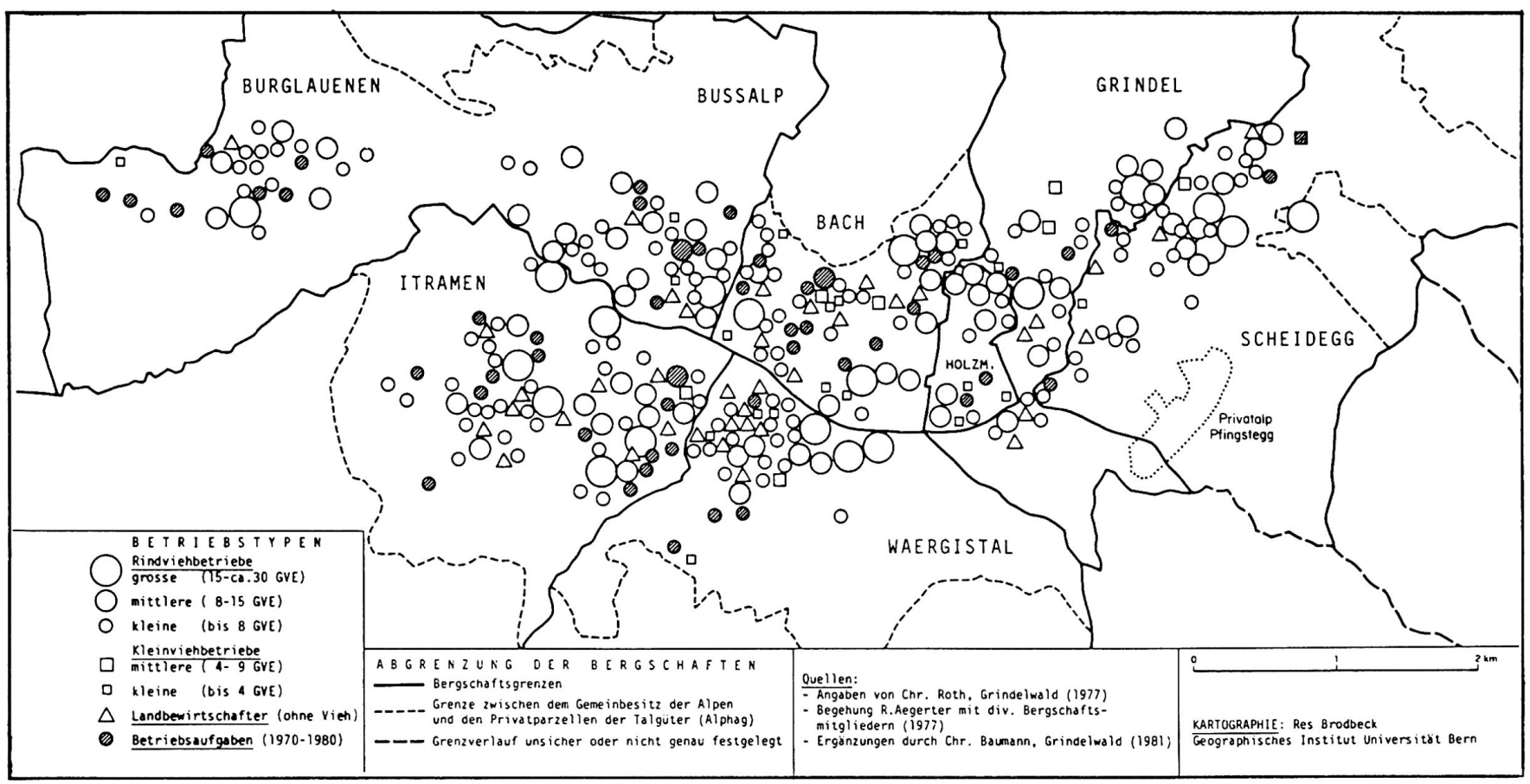

Quelle: Eigene Ergebungen

Erläuterungen: Bei den "Bergschaften" handelt es sich um Gemeindefraktionen, welche jeweils alle diejenigen

Talgutparzellen umfassen, die mit den Kuhrechten einer bestimmten Genossenschaftsalp "geseyt" sind.

Abb. 1 Standorte der landwirtschaftlichen Betriebe in Grindelwald 
sich akzentuierende interne Umschichtung bzw. Polarisierung der Betriebsstruktur (vgl. Abb. 2):

Geschrumpft ist im Zeitraum 1970-1980 vor allem die Zahl der kleinen Rindviehbetriebe, gehen doch $92 \%$ der Betriebsaufgaben auf deren Konto. Die verbleibenden Betriebe dieses Typs, welche allerdings immer noch $46 \%$ aller Betriebe ausmachen, sowie die Kleinviehbetriebe haben in ihrer Entwicklung mehrheitlich stagniert. Kräftig expandiert haben andererseits im gleichen Zeitraum die meisten der heutigen Großbetriebe sowie etwas weniger als die Hälfte der mittleren Betriebe. Wie die neusten Zahlen für 1985 zeigen, hält dieser Trend in etwas abgeschwächtem Ausmaß auch in den 1980er Jahren weiter an.

\section{Einige Ursachen und vermutete Folgen}

Die Ursachen und Folgen dieses grob skizzierten landwirtschaftlichen Strukturwandels sind vielschichtig und können hier nicht ausführlich abgehandelt werden. Einige Hinweise müssen genügen.

\section{Zu den Ursachen}

Was die Verursachung anbelangt, so ist dieser betriebliche Strukturwandel in erster Linie als ein $A n$ passungsproze $\beta$ der Landwirtschaft an unsere wachsende industrie- und dienstleistungsorientierte Volkswirtschaft zu sehen. Ein genereller sozialökonomischer Wandel also, welcher unser Berggebiet etwa gegen Ende der 1960er Jahre erfaßt hat - mit einer gewissen zeitlichen Verzögerung gegenüber dem Mittelland -, und welcher bis heute noch nicht abgeschlossen ist. Im Rahmen dieses Wandels transformiert sich die ehemals auf Autarkie ausgerichtete Berglandwirtschaft immer mehr zu einem Wirtschaftszweig, welcher nur noch auf der Basis einer breiten finanziellen Abstützung auf außerlandwirtschaftliche Erwerbsquellen sowie staatliche Transferleistungen überlebensfähig bleibt .

Im Falle der touristisch stark entwickelten $\mathrm{Ge}$ meinde Grindelwald hat sich im weiteren gezeigt, da $\beta$ dieser allgemeine Anpassungsproze $\beta$ durch die besondere endogene Situation nach zwei Seiten hin modifiziert wird:

Die touristische Entwicklung hat einerseits zum Aufbau von Großbetrieben mit beigetragen, indem es deren Betriebsleiter verstanden haben, zusätzlich zur Landwirtschaft bereits recht frühzeitig ein zweites «wirtschaftliches Bein» im Tourismussektor zu verankern, sei dies als Detailhändler, Baugewerbler, Hotelier oder Restaurantbetreiber. Die hierbei erzielten Einkommen haben es dann in vielen Fällen ermöglicht, den Betrieb auszuweiten, großzügig Maschinen anzuschaffen und die dezentralisierten Betriebsgebäude zu sanieren.
Auf der anderen Seite hat aber dieselbe touristische Entwicklung einen vielseitigen Arbeitsmarkt geschaffen, welcher den Landwirten mit kleineren und mittleren Betrieben alle Arten von saisonalen oder ganzjährigen, teil- oder ganzzeitlichen Erwerbskombinationen erlaubt (vgl. hierzu auch WIESMANN 1986). Dies wiederum hat ganz wesentlich zur Beharrung einer überproportionalen Anzahl von Kleinbetrieben beigetragen, welche einer weiteren Expansion der Großbetriebe nun teilweise im Wege stehen.

\section{Zu den Folgen}

Was nun die Folgen dieses betrieblichen Strukturwandels anbelangt, so lassen sich diese auf verschiedenen Ebenen ausmachen:

Auf der Ebene der Landwirtschaft als Wirtschaftszweig hat unzweifelhaft eine gewisse Stärkung der Betriebsstruktur stattgefunden, allerdings auf Kosten einer erhöhten finanziellen Abhängigkeit vom Dienstleistungssektor und der öffentlichen Hand.

Auch auf der Ebene des räumlichen Nutzungssystems hat sich bisher der Strukturwandel nicht merklich negativ ausgewirkt. Allerdings besteht eine gewisse Tendenz zur räumlichen Nutzungspolarisierung, indem gut erschlossene und mechanisch bewirtschaftbare landwirtschaftliche Vorrangflächen zunehmend intensiver bewirtschaftet und periphere Grenzertragsstandorte nur noch beweidet werden. Die Tatsache, daß während der Felderhebungszeit Anfang der 1980er Jahre in Grindelwald nur wenig Brachland ausgemacht werden konnte, beruht v. a. darauf, daß derzeit viele dieser Grenzertragsflächen noch von traditionell ausgerichteten mittelgroBen Betrieben sowie vor allem von Klein- und Kleinviehbetrieben bewirtschaftet werden. Bei einem weiteren Rückgang dieser mittleren und kleinen Betriebe besteht demnach die Gefahr, daß die flächendeckende Nutzung des landwirtschaftlichen Bodens nicht mehr gewährleistet werden könnte. Dies wiederum hätte beträchtliche Folgen für das Landschaftsbild und die natürliche Umwelt (vgl. MESSERLI u. a. 1986).

Als eine für die Landwirtschaft nicht ganz unproblematische Folge des sozialökonomischen Wandels zeichnet sich allerdings ein Prozeß einer doppelten räumlichen Verlagerung der Betriebsstandorte ab: Wie die Abbildung 3 veranschaulicht, ziehen sich einerseits seit längerer Zeit die Betriebe im ganzen Gemeindegebiet aus der Peripherie in Richtung Talgrund/Dorf zurück. Andererseits zeichnet sich ein aktueller Verdrängungsproze $\beta$ aus dem zunehmend touristisch erschlossenen Dorfzentrum resp. der umgebenden Bauzone ${ }^{3}$ ab. Die Folge ist eine wachsende Konzentration der Betriebsstandorte auf einen dorfnahen Ring von landwirtschaftlichen Gunstflächen; ein Prozeß, welcher zu einer weite- 
$a$

\begin{tabular}{|c|c|c|c|c|c|c|c|c|c|c|c|c|c|c|}
\hline \multicolumn{2}{|c|}{$\begin{array}{l}\text { Betriebs- } \\
\text { Typen }\end{array}$} & \multicolumn{2}{|c|}{$\begin{array}{l}\begin{array}{l}\text { Expandierende } \\
\text { Betriebe }\end{array} \\
\text { abs. } \quad \%\end{array}$} & \multicolumn{2}{|c|}{$\begin{array}{l}\text { Stagnierende } \\
\text { Betriebe }\end{array}$} & \multicolumn{2}{|c|}{$\begin{array}{l}\text { Reduzierende } \\
\text { Betriebe }\end{array}$} & \multicolumn{2}{|c|}{$\begin{array}{l}\text { Landbewirt- } \\
\text { schaftung }\end{array}$} & \multicolumn{2}{|c|}{$\begin{array}{l}\text { Total Betriebe } \\
\quad 1980\end{array}$} & \multicolumn{2}{|c|}{$\begin{array}{l}\text { Betriebs- } \\
\text { aufgaben } \\
\text { 1970-80 }\end{array}$} & $\begin{array}{l}\text { Total Betriebe } \\
\quad 1985\end{array}$ \\
\hline \multirow{3}{*}{ 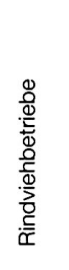 } & $\begin{array}{l}\text { Grossbetriebe } \\
(15,1-c a .30 \text { GVE) }\end{array}$ & 18 & 90 & 2 & 10 & - & - & - & - & 20 & $\left(\begin{array}{l}100 \% \\
(7 \%)\end{array}\right.$ & - & - & 19 \\
\hline & $\begin{array}{l}\text { mittlere Betriebe } \\
(8,1-15 \mathrm{GVE})\end{array}$ & 26 & 40 & 36 & 55 & 3 & 5 & - & - & 65 & $\begin{array}{l}100 \% \\
(23 \%)\end{array}$ & 3 & 6 & 67 \\
\hline & $\begin{array}{l}\text { Kleinbetriebe } \\
\text { (bis } 8 \text { GVE) }\end{array}$ & 5 & 4 & 112 & 85 & 14 & 11 & - & - & 131 & $\begin{array}{l}100 \% \\
(46 \%)\end{array}$ & 47 & 92 & 123 \\
\hline \multirow{2}{*}{ : } & $\begin{array}{l}\text { mittlere Kleinviehbetriebe } \\
(4,1-\text { ca. } 8 \text { GVE) }\end{array}$ & 1 & - & 6 & 86 & - & - & - & - & 7 & $\left(\begin{array}{l}100 \% \\
(3 \%)\end{array}\right.$ & - & - & 10 \\
\hline & $\begin{array}{l}\text { Kleine Kleinviehbetriebe } \\
\text { (bis } 4 \text { GVE) }\end{array}$ & - & - & 18 & 95 & 1 & 5 & - & - & 19 & $\begin{array}{l}100 \% \\
(7 \%)\end{array}$ & 1 & 2 & 13 \\
\hline \multicolumn{2}{|c|}{$\begin{array}{l}\text { Landbewirtschafter und Spezialbetriebe } 1) \\
(0,25-\text { ca. } 2 \text { ha) }\end{array}$} & - & - & - & - & - & - & 40 & 100 & 40 & $\begin{array}{l}100 \% \\
(14 \%)\end{array}$ & $?$ & & $?$ \\
\hline \multicolumn{2}{|c|}{ TOTAL BETRIEBE 1980} & 53 & 19 & 171 & 61 & 18 & 6 & 40 & 14 & 282 & $\begin{array}{l}100 \% \\
(100 \%)\end{array}$ & 51 & 100 & $246^{2)}$ \\
\hline \multicolumn{2}{|c|}{$\begin{array}{l}\text { NUR VIEHHALTEBETRIEBE } \\
100 \%=242\end{array}$} & & $22 \%$ & & $71 \%$ & & $7 \%$ & & & & & & & \\
\hline
\end{tabular}

Quellen: Aufnahmeprotokolle der “Kostenbeiträge an Viehhalter im Berggebiet und in der voralpinen Hügelzone” von 1970 bis 1980 und 1985

Erläuterungen: "Als "Landbewirtschafter" bezeichne ich eine Gruppe kleinster Übergangsbetriebe ohne eigentliche Tierhaltung, welche nur noch einige Parzellen pflegen. Unter "Spezialbetriebe" fallen 3 Halter von Reitpferden sowie 2 Hühnerbetriebe 2) Ohne die "Landbewirtschafter" und "Spezialbetriebe"

Abb. 2 Entwicklungsdynamik der landwirtschaftlichen Betriebe im Zeitraum 1970-80/85 (basierend auf der Veränderung des GVE-Bestandes) 
Abb. 3 Räumlich-zeitliche Verschiebungstendenzen der landwirtschaftlichen Betriebsstandorte in Grindelwald

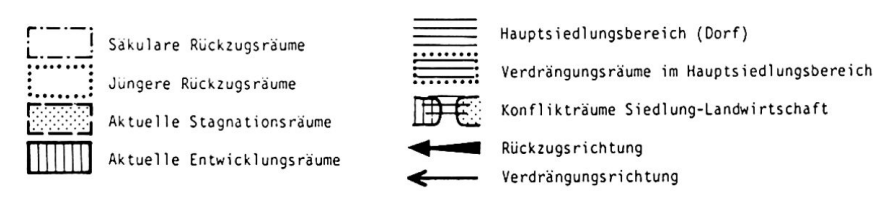

QUELLEN: Xostenbeitröge an Viehh. im Bergg., AEGERTER 1983, eigene Erhebungen

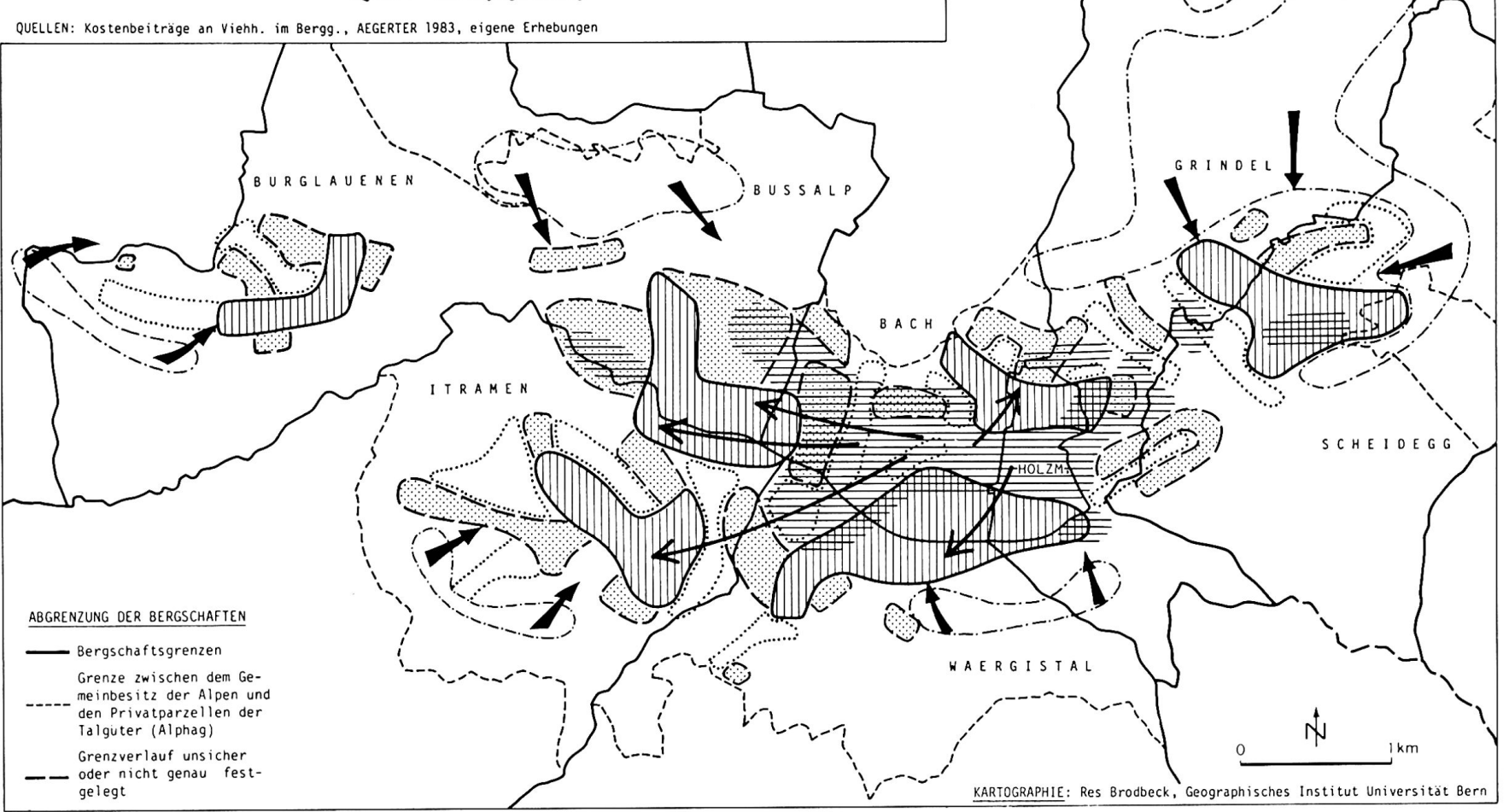


ren Nutzungsintensivierung auf diesen Flächen, zu Flächenkonflikten mit der touristischen Siedlung und damit zu einem wachsenden Druck auf den in dieser Zone ohnehin bereits sehr angespannten Bodenmarkt führt.

Diese Ausgangslage des betrieblichen Strukturwandels, interpretiert als ein kurz- bis mittelfristiger Ausschnitt eines längerfristigen und generelleren sozialökonomischen Wandels der Landwirtschaft in einer touristisch stark entwickelten Berggemeinde, gibt nun den Rahmen ab für die im folgenden Teil vorzustellende Grundeigentumserhebung.

\section{Landwirtschaftliche Grundeigentumsstruktur als Abbild des sozialökonomischen Wandels}

Eine Erfassung der landwirtschaftlichen Grundeigentumsverhältnisse kann von verschiedenen Fragestellungen ausgehen. Im Vordergrund gestanden hat bei unserer Untersuchung weniger die direkte Kopplung der Eigentumsverhältnisse an die betriebsstrukturelle Situation als die Frage, inwiefern sich der vorangehend geschilderte sozialökonomische Wandel der Landwirtschaft heute bereits in der Grundeigentumsstruktur niederschlägt.

Hinter dieser Frage verbirgt sich nämlich die schon mehrfach sowohl von Geographen wie auch von Agrarsoziologen geäußerte These, daß die Grundeigentumsstruktur als ein sozialräumliches Ordnungsnetz aufgefaßt werden kann, dessen rahmengebende Funktion weit über die nutzungs- und verfügungsrechtliche Ebene hinausreicht (vgl. etwa GALLUSSER 1979, PLANCK und ZICHE 1979, BOEHM 1980).

Boden bzw. Grundeigentum bedeutet nämlich nicht nur Verfügungsgewalt über einen Produktionsfaktor, sondern verhilft gerade in der ländlichen Gesellschaft auch zu sozialem Rang (vgl. MEYRATSCHLEE 1983). Da sich zudem ein ganz wesentlicher Teil der alltäglichen Aktivitäten und sozialen Beziehungen der ländlichen Bevölkerung im Rahmen der räumlichen Besitzordnungsmuster abspielt (wie etwa tägliche Arbeitsabläufe, nachbarschaftliche Kontakte usw.), kann man sogar davon ausgehen, daß den Grundeigentumsverhältnissen in ländlichen Sozialsystemen eine wesentliche Steuerungsfunktion zukommt. ${ }^{+}$

Wenn diese These zutrifft, könnte die Grundeigentumsstruktur somit als eine Art verräumlichter «Makroindikator» für die Transformation einer ehemaligen Agrargesellschaft zu einer zunehmenden Dienstleistungsgesellschaft angesehen werden.

Im Rahmen der Landwirtschaftsuntersuchung im MAB-Testgebiet Grindelwald ist es allerdings nicht möglich gewesen, diese angedeuteten Zusammen- hänge zwischen Grundeigentumsstruktur, Bodennutzung, sozialer Ordnung und individueller Betriebsentwicklung in voller Tiefe auszuloten. Dies unter anderem darum, weil bereits die Erstellung einer flächendeckenden Grundeigentumskarte und deren statistische Auswertung einen außerordentlich großen Arbeitaufwand erfordert haben.5

Was im folgenden vorgestellt wird, kann deshalb nicht mehr sein als ein erster, inventarisierender Schritt zu einer umfassenderen Grundeigentumsanalyse.

Als eine weitere Präzisierung ist zudem darauf hinzuweisen, daß nur die landwirtschaftlich nutzbaren Parzellen in die Erhebung einbezogen worden sind. Ausgeschlossen blieben demnach die mehrheitlich überbauten Flächen (diese umfassen rund 120 ha innerhalb und rund 55 ha außerhalb der Bauzone, bzw. zusammen rund $5 \%$ der parzellierten Talgutfläche Grindelwalds von total $3558 \mathrm{ha}$ ), die größeren Verkehrs- und Sportflächen (wie Parkplätze, Eisenbahngelände und Tennisplätze) sowie einige private Waldparzellen.

\section{Differenzierung nach sozialökonomischen Eigentümergruppen}

Zur detaillierten Sichtbarmachung des weiter vorne angesprochenen sozialökonomischen Wandels erschien es sinnvoll, eine relativ fein gegliederte Gruppierung der Eigentümer vorzunehmen (vgl. die 19 Eigentümerkategorien in Abb. 4). Aus kartographischen und finanziellen Gründen mußte diese Gliederung für die gedruckte Farbkarte jedoch auf 7 Kategorien komprimiert werden (vgl. die beigelegte Falzkarte "MAB-Projekt Grindelwald: Grundeigentumsverhältnisse der landwirtschaftlichen Parzellen»).

Eine Auswertung der Grundeigentumsstatistik in Abb. 4 zeigt, daß die Landwirtschaft im weiten Sinne (Kategorien 3-9) in Grindelwald heute noch gut $73 \%$ des von ihr bewirtschafteten Bodens in eigenen Händen hält. Die restlichen $27 \%$ sind in den zweiten und vor allem dritten Sektor abgewandert. Eingeschränkt auf die eigentlichen aktiven Landwirtschaftsbetriebe (Kat. 3, 6, 8, 9) macht ihr Anteil allerdings nur noch rund $55 \%$ aus.

Der Anteil des öffentlichen Sektors (Kat. 1, 2, 17) ist mit nur $0,6 \%$ erwartungsgemäß sehr klein. Eher hoch erscheint mit $8,2 \%$ jedoch der Anteil der nicht mehr im Erwerbsleben stehenden Rentner. Der Anteil der (unverteilten) Erbengemeinschaften (Kat. $5,15,18$ ) hält sich mit $4,1 \%$ für ein ländliches Gebiet in Grenzen. Auffallend ist hingegen, daß $9 \%$ der landwirtschaftlichen Parzellen in Händen von Auswärtigen (Kat. 16, 17, 18, 19) liegen (unter diesen finden sich natürlich auch noch etliche ausgewanderte Grindelwalder). 


\section{Differenzierung nach der Betriebsstruktur}

Hinsichtlich der Zusammenhänge zwischen der Grundeigentumsstruktur und der Betriebsstruktur lassen sich aus Abbildung 4 grob die folgenden Tendenzen ermitteln:

Mit steigender Betriebsgröße nimmt der Anteil der Betriebsfläche, welcher sich aus Grundeigentum in Händen der Landwirtschaft zusammensetzt, tendenziell ab (von rund $75 \%$ bei Kleinviehbetrieben auf $62 \%$ bei den Großbetrieben). Der Anteil in Händen des zweiten und dritten Sektors nimmt hingegen entsprechend zu. Diese Tendenz würde noch stärker ausfallen, wenn man das Grundeigentum der «Hotellandwirte» und "Geschäftslandwirte», welches in dieser Betrachtung ganz zur Landwirtschaft gerechnet worden ist, anteilig auf die drei Sektoren verteilen würde.

Anders stellt sich die Situation jedoch bei den Landbewirtschaftern dar: Der Flächenanteil in Händen der Landwirtschaft beträgt hier nur noch rund $39 \%$, derjenige des zweiten und dritten Sektors jedoch bereits an die $50 \%$. Dies ist insofern nicht erstaunlich, als der größere Teil der Landbewirtschafter ja bereits in Berufen dieser beiden Sektoren tätig ist.

\section{Räumliche Differenzierung}

Eine überblickmäßige räumliche Analyse der Grundeigentumsstruktur (vgl. die beigelegte Falzkarte) gibt deutliche Hinweise darauf, daß im Rahmen des langfristigen sozialökonomischen Wandels offensichtlich auch eine gewisse räumliche Differenzierung der Eigentumsverhältnisse vor sich geht.

Sehr deutlich ist zum Beispiel ein Entwicklungsgegensatz zu erkennen zwischen dem zentralen Dorfbereich, wo bereits eine starke Durchmischung der Eigentumsverhältnisse stattgefunden hat, und den peripheren Gemeindeteilen. So überwiegt etwa in den Bergschaften Itramen und Burglauenen oder im nordöstlichen Bereich von Grindel das landwirtschaftliche Grundeigentum noch bei weitem, und auch die durchschnittlichen Parzellenflächen sind noch bedeutend größer als in der begehrten Dorflage.

Etwas weniger deutlich, aber visuell doch erkennbar, finden wir im weiteren eine gewisse Konzentration der Parzellen von Altlandwirten, Witwen und Rentnern in eher abseitigen Lagen wie etwa im südwestlichen Bereich der Bergschaft Burglauenen, im mittleren bis oberen Teil von Wärigstal, sowie im mittleren und oberen Teil von Grindel und Scheidegg.

Hierbei handelt es sich in der Regel um Parzellen auslaufender oder bereits aufgegebener Altersbetriebe, welche wegen ihrer ungünstigen Lage nicht mehr weitergeführt werden. Demgegenüber konzentrieren sich die Parzellen der "Hotel- und Ge- schäftslandwirte», welche zumeist mittlere bis große Landwirtschaftsbetriebe führen, zunehmend auf gut bewirtschaftbare Tallagen bzw. ebensolche Vorsassen. Diese Polarisierung muß demnach als Anzeichen eines sich innerhalb der Landwirtschaft abspielenden räumlichen Verdrängungswettbewerbs gesehen werden.

Die Parzellen der Gewerbe- und Handeltreibenden zeigen keine bestimmbare räumliche Verteilungstendenz auf. Hingegen fällt auf, daß die Gruppe $\mathrm{Ho}$ tellerie und Gastgewerbe noch ziemlich viel landwirtschaftliches Grundeigentum in der dorfnahen Talgutstufe und in guter Vorsassenlage besitzt. ${ }^{6}$ Dieses Eigentum stammt in einigen Fällen aus der Zeit, als einige dieser Hoteliers ebenfalls noch einen Landwirtschaftsbetrieb geführt haben. In anderen Fällen mag es sich auch um neuere Grundeigentumskäufe handeln.

Ein deutliches räumliches Verteilungsmuster läßt sich schließlich auch bei den Parzellen der Auswärtigen erkennen. Diese Flächen konzentrieren sich auf gute, südexponierte Lagen vor allem in den dorfnahen Bergschaften Bach, Grindel, Holzmatten und Bußalp, sowie auf einige Vorsassen in den Bergschaften Grindel, Itramen und Bußalp.

\section{Bilanz und Ausblick}

Die vorangehend ausgebreiteten Untersuchungsergebnisse lassen erkennen, daß die Landwirtschaft in Grindelwald einen spezifischen betriebsstrukturellen, nutzungs- und eigentumsräumlichen Transformationsproze $\beta$ durchmacht. Dieser Prozeß kann verallgemeinernd durch eine zunehmende Verlagerung der Verfügungsgewalt über die sektorspezifischen Produktionsmittel in den Gewerbe- und Dienstleistungssektor gekennzeichnet werden.

Obschon die landwirtschaftliche Nutzung zurzeit nach außen noch relativ intakt erscheint und auch die landwirtschaftliche Bevölkerung in Grindelwald immer noch ein beträchtliches politisches und soziales Ansehen genießt, besteht eine gewisse Gefahr, daß im Rahmen dieses schleichenden Wandels die ehemals dominante landwirtschaftliche Bevölkerungsgruppe zunehmend die Kontrolle über ihren ursprünglichen Wirtschafts- und Lebensraum verliert (vgl. hierzu auch MESSERLI u.a. 1986: 50-58).

Man kann diesen Prozeß von «außen» als eine folgerichtige «Sukzessionserscheinung» im Rahmen der touristischen Entwicklung unseres Alpenraumes betrachten (LICHTENBERGER 1979). Von «innen» wird dieser Sukzessionsvorgang für die Betroffenen allerdings zur schmerzlichen Erfahrung, beinahe tagtäglich erleben zu müssen, wie man von einer neuen Dienstleistungs- und Freizeitgesellschaft mit neuen Lebensformen, neuen Wirtschaftlichkeits- 
Abb. 4 Verteilung der landwirtschaftl. genutzten Parzellen nach Eigentümerkategorien und Betriebstypen

\begin{tabular}{|c|c|c|c|c|c|c|c|c|c|c|c|}
\hline \multirow[b]{2}{*}{$\begin{array}{l}\text { Grund- } \\
\text { Eigentümer }\end{array}$} & \multicolumn{3}{|c|}{ Rindviehbetriebe } & \multicolumn{2}{|c|}{ Kleinviehbetriebe } & \multirow{2}{*}{\begin{tabular}{|c|} 
Landbe- \\
wirt- \\
schafter \\
Typ 6 \\
\end{tabular}} & \multirow{2}{*}{$\begin{array}{l}\text { Totale } \\
\text { Anz. } \\
\text { Parz. }\end{array}$} & \multirow{2}{*}{$\begin{array}{c}\text { Anteil } \\
\text { aller } \\
\text { Parz. } \\
(\%)\end{array}$} & \multirow{2}{*}{$\begin{array}{c}\varnothing \text { Parz. } \\
\text { Flä- } \\
\text { che } \\
\text { (ha) }\end{array}$} & \multirow{2}{*}{$\begin{array}{l}\text { Totale } \\
\text { Flä- } \\
\text { che } \\
\text { (ha) }\end{array}$} & \multirow{2}{*}{$\begin{array}{l}\text { Ant. an } \\
\text { der } \\
\text { Ges. FI. } \\
(\%)\end{array}$} \\
\hline & $\begin{array}{l}\text { Grosse } \\
\text { Typ } 1 \\
\end{array}$ & $\begin{array}{l}\text { Mittlere } \\
\text { Typ } 2 \\
\end{array}$ & $\begin{array}{l}\text { Kleine } \\
\text { Typ } 3 \\
\end{array}$ & $\begin{array}{c}\text { Mittlere } \\
\text { Typ } 4 \\
\end{array}$ & $\begin{array}{l}\text { Kleine } \\
\text { Typ } 5 \\
\end{array}$ & & & & & & \\
\hline $\begin{array}{l}\text { 1. Einwohnergemeinde } \\
\text { Grindelwald }\end{array}$ & $\begin{array}{c}1 \\
25,0^{11} \\
0,4 \\
0,1 \\
\end{array}$ & $\begin{array}{r}1 \\
25,0 \\
0,2 \\
0,1 \\
\end{array}$ & $\begin{array}{l}1 \\
25,0 \\
0,2 \\
0,1 \\
\end{array}$ & $\begin{array}{l}0 \\
0 \\
0 \\
0\end{array}$ & $\begin{array}{l}0 \\
0 \\
0 \\
0\end{array}$ & $\begin{array}{r}1 \\
25,0 \\
1,8 \\
0,1 \\
\end{array}$ & 4 & 0,2 & 0,36 & 1,5 & 0,1 \\
\hline $\begin{array}{l}\text { 2. Bergschaften und } \\
\text { einh. Vereine }\end{array}$ & $\begin{array}{r}2 \\
40,0 \\
0,8 \\
0,1 \\
\end{array}$ & $\begin{array}{r}2 \\
40,0 \\
0,4 \\
0,1 \\
\end{array}$ & $\begin{array}{r}1 \\
20,0 \\
0,2 \\
0,1 \\
\end{array}$ & $\begin{array}{l}0 \\
0 \\
0 \\
0 \\
\end{array}$ & $\begin{array}{l}0 \\
0 \\
0 \\
0 \\
\end{array}$ & $\begin{array}{l}0 \\
0 \\
0 \\
0 \\
\end{array}$ & 5 & 0,3 & 1,00 & 5,1 & 0,3 \\
\hline $\begin{array}{l}\text { 3. aktive Landwirte } \\
\text { (inkl. Frauengut; } \\
\text { z. T. nur Mit- oder } \\
\text { Gesamteig.anteile) }\end{array}$ & $\begin{array}{r}61 \\
8,9 \\
24,0 \\
3,5 \\
\end{array}$ & $\begin{array}{c}230 \\
33,4 \\
41,2 \\
13,1 \\
\end{array}$ & \begin{tabular}{r|}
338 \\
49,1 \\
51,0 \\
19,3 \\
\end{tabular} & $\begin{array}{r}17 \\
2,5 \\
47,2 \\
1,0 \\
\end{array}$ & $\begin{array}{r}30 \\
4,4 \\
42,9 \\
1,7 \\
\end{array}$ & $\begin{array}{r}12 \\
1,7 \\
21,1 \\
0,7 \\
\end{array}$ & 689 & 42,1 & 0,99 & 679,2 & 45,2 \\
\hline $\begin{array}{l}\text { 4. Fam.angehörige od. } \\
\text { enge Verwandte } \\
\text { aktiver Landwirte } \\
\text { (Eltern, Schwieger- } \\
\text { eltern, Kinder, } \\
\text { Geschwister) } \\
\end{array}$ & $\begin{array}{l}8 \\
5,5 \\
3,1 \\
0,5\end{array}$ & $\begin{array}{r}48 \\
32,9 \\
8,6 \\
2,7\end{array}$ & $\begin{array}{c}77 \\
52,7 \\
11,6 \\
4,4\end{array}$ & $\begin{array}{r}6 \\
4,1 \\
16,7 \\
0,3\end{array}$ & $\begin{array}{l}2 \\
1,4 \\
2,9 \\
0,1\end{array}$ & $\begin{array}{l}5 \\
3,4 \\
8,9 \\
0,3\end{array}$ & 146 & 8,9 & 1,00 & 146,2 & 9,7 \\
\hline $\begin{array}{l}\text { 5. "landw.» Erbengem. } \\
\text { (mind. ein Miterbe } \\
\text { od. dessen Ehe- } \\
\text { partner führt den B.) }\end{array}$ & $\begin{array}{r}3 \\
11,1 \\
1,2 \\
0,2 \\
\end{array}$ & $\begin{array}{r}10 \\
37,0 \\
1,8 \\
0,6 \\
\end{array}$ & $\begin{array}{l}11 \\
40,7 \\
1,7 \\
0,6 \\
\end{array}$ & $\begin{array}{l}0 \\
0 \\
0 \\
0\end{array}$ & $\begin{array}{l}2 \\
7,4 \\
2,9 \\
0,1\end{array}$ & $\begin{array}{l}1 \\
3,7 \\
1,8 \\
0,1 \\
\end{array}$ & 27 & 1,6 & 1,03 & 27,8 & 1,8 \\
\hline $\begin{array}{l}\text { 6. aktive Landwirte } \\
\text { aus der Gemeinde } \\
\text { Lütschental }\end{array}$ & $\begin{array}{r}1 \\
33,3 \\
0,4 \\
0,1 \\
\end{array}$ & $\begin{array}{l}0 \\
0 \\
0 \\
0\end{array}$ & $\begin{array}{l}1 \\
33,3 \\
0,2 \\
0,1 \\
\end{array}$ & $\begin{array}{l}0 \\
0 \\
0 \\
0 \\
\end{array}$ & $\begin{array}{l}0 \\
0 \\
0 \\
0 \\
\end{array}$ & $\begin{array}{l}0 \\
0 \\
0 \\
0\end{array}$ & 2) & 0,1 & 0,96 & $2,9^{2)}$ & 0,2 \\
\hline $\begin{array}{l}\text { 7. Altlandw., Witwen } \\
\text { von Lw., div. Rentner } \\
\text { u. Witw. mit nicht } \\
\text { rekonstr. Berufen } \\
\text { (älter als 1915) }\end{array}$ & $\begin{array}{r}15 \\
15,8 \\
5,9 \\
0,9\end{array}$ & $\begin{array}{r}39 \\
41,1 \\
7,0 \\
2,2\end{array}$ & $\begin{array}{l}33 \\
34,7 \\
5,0 \\
1,9\end{array}$ & $\begin{array}{l}2 \\
2,1 \\
5,6 \\
0,1\end{array}$ & $\begin{array}{l}3 \\
3,2 \\
4,3 \\
0,2\end{array}$ & $\begin{array}{l}3 \\
3,2 \\
5,3 \\
0,2\end{array}$ & 95 & 5,8 & 0,89 & 84,1 & 5,6 \\
\hline 8. "Hotellandwirte" & $\begin{array}{r}43 \\
65,2 \\
16,9 \\
2,5 \\
\end{array}$ & $\begin{array}{r}7 \\
10,6 \\
1,3 \\
0,4 \\
\end{array}$ & $\begin{array}{c}7 \\
10,6 \\
1,1 \\
0,4 \\
\end{array}$ & $\begin{array}{l}1 \\
1,5 \\
2,8 \\
0,1\end{array}$ & $\begin{array}{r}8 \\
12,1 \\
11,4 \\
0,5 \\
\end{array}$ & $\begin{array}{l}0 \\
0 \\
0 \\
0\end{array}$ & 66 & 4,0 & 1,02 & 67,8 & 4,5 \\
\hline $\begin{array}{l}\text { 9. "Geschäftsland- } \\
\text { wirte" }\end{array}$ & $\begin{array}{r}28 \\
33,7 \\
11,0 \\
1,6\end{array}$ & $\begin{array}{r}30 \\
36,1 \\
5,4 \\
1,7\end{array}$ & \begin{tabular}{l|}
16 \\
19,3 \\
2,4 \\
0,9
\end{tabular} & $\begin{array}{l}1 \\
1,2 \\
2,8 \\
0,1\end{array}$ & $\begin{array}{r}7 \\
8,4 \\
10,0 \\
0,4 \\
\end{array}$ & $\begin{array}{l}1 \\
1,2 \\
1,8 \\
0,1\end{array}$ & 83 & 5,1 & 1,05 & 87,6 & 5,8 \\
\hline $\begin{array}{l}\text { 10. Gewerbe und Handel } \\
\text { (Selbständige und } \\
\text { Angestellte) }\end{array}$ & $\begin{array}{r}13 \\
17,3 \\
5,1 \\
0,7 \\
\end{array}$ & $\begin{array}{r}27 \\
36,0 \\
4,8 \\
1,5 \\
\end{array}$ & $\begin{array}{l}21 \\
28,0 \\
3,2 \\
1,2 \\
\end{array}$ & $\begin{array}{l}1 \\
1,3 \\
2,8 \\
0,1 \\
\end{array}$ & $\begin{array}{l}2 \\
2,7 \\
2,9 \\
0,1\end{array}$ & $\begin{array}{r}11 \\
14,7 \\
19,3 \\
0,6 \\
\end{array}$ & 75 & 4,6 & 0,75 & 56,6 & 3,8 \\
\hline $\begin{array}{l}\text { 11. Hotellerie und } \\
\text { Gastgewerbe (v. a. } \\
\text { Selbständige) }\end{array}$ & $\begin{array}{c}5 \\
11,6 \\
2,0 \\
0,3\end{array}$ & $\begin{array}{r}22 \\
51,2 \\
3,9 \\
1,3 \\
\end{array}$ & $\begin{array}{l}14 \\
32,6 \\
2,1 \\
0,8 \\
\end{array}$ & $\begin{array}{l}0 \\
0 \\
0 \\
0\end{array}$ & $\begin{array}{l}0 \\
0 \\
0 \\
0\end{array}$ & $\begin{array}{l}2 \\
4,7 \\
3,5 \\
0,1\end{array}$ & 43 & 2,6 & 0,93 & 40,2 & 2,7 \\
\hline $\begin{array}{l}\text { 12. Verkehrs- und } \\
\text { Transportbetriebe }\end{array}$ & $\begin{array}{l}0 \\
0 \\
0 \\
0\end{array}$ & $\begin{array}{c}7 \\
53,8 \\
1,3 \\
0,4 \\
\end{array}$ & \begin{tabular}{l}
\multicolumn{1}{c}{6} \\
46,2 \\
0,9 \\
0,3 \\
\end{tabular} & $\begin{array}{l}0 \\
0 \\
0 \\
0 \\
\end{array}$ & $\begin{array}{l}0 \\
0 \\
0 \\
0\end{array}$ & $\begin{array}{l}0 \\
0 \\
0 \\
0 \\
\end{array}$ & 13 & 0,8 & 0,53 & 6,8 & 0,4 \\
\hline
\end{tabular}




\begin{tabular}{|c|c|c|c|c|c|c|c|c|c|c|c|}
\hline \multirow[b]{2}{*}{$\begin{array}{l}\text { Grund- } \\
\text { Eigentümer }\end{array}$} & \multicolumn{3}{|c|}{ Rindviehbetriebe } & \multicolumn{2}{|c|}{ Kleinviehbetriebe } & \multirow{2}{*}{$\begin{array}{c}\text { Landbe- } \\
\text { wirt- } \\
\text { schafter } \\
\text { Typ 6 }\end{array}$} & \multirow{2}{*}{$\begin{array}{l}\text { Totale } \\
\text { Anz. } \\
\text { Parz. }\end{array}$} & \multirow{2}{*}{$\begin{array}{l}\text { Anteil } \\
\text { aller } \\
\text { Parz. } \\
(\%)\end{array}$} & \multirow{2}{*}{$\begin{array}{c}\varnothing \text { Parz. } \\
\text { Flä- } \\
\text { che } \\
\text { (ha) }\end{array}$} & \multirow{2}{*}{$\begin{array}{l}\text { Totale } \\
\text { Flä- } \\
\text { che } \\
\text { (ha) }\end{array}$} & \multirow{2}{*}{$\begin{array}{l}\text { Ant. an } \\
\text { der } \\
\text { Ges. FI. } \\
(\%)\end{array}$} \\
\hline & Grosse & $\begin{array}{c}\text { Mittlere } \\
\text { Typ } 2 \\
\end{array}$ & Kleine & $\begin{array}{c}\text { Mittlere } \\
\text { Typ } 4 \\
\end{array}$ & $\begin{array}{l}\text { Kleine } \\
\text { Typ } 5 \\
\end{array}$ & & & & & & \\
\hline $\begin{array}{l}\text { 13. Übr. Dienstl.bereich } \\
\text { (Selbst. u. Angest. } \\
\text { wie Skilehr., Bergf. } \\
\text { Chauff., Bahnang., } \\
\text { Brieftr., Arzt, } \\
\text { Lehrer usw.) }\end{array}$ & $\begin{array}{r}24 \\
23,5 \\
9,4 \\
1,4\end{array}$ & $\begin{array}{r}38 \\
37,3 \\
6,8 \\
2,2\end{array}$ & $\begin{array}{l}23 \\
22,5 \\
3,5 \\
1,3\end{array}$ & $\begin{array}{l}2 \\
2,0 \\
5,6 \\
0,1\end{array}$ & $\begin{array}{r}7 \\
6,9 \\
10,0 \\
0,4\end{array}$ & $\begin{array}{r}7 \\
6,9 \\
12,3 \\
0,4\end{array}$ & 102 & 6,2 & 0,88 & 89,9 & 6,0 \\
\hline $\begin{array}{l}\text { 14. nichtlandwirt. } \\
\text { Rentner (älter als } \\
\text { 1915) }\end{array}$ & $\begin{array}{r}9 \\
16,7 \\
3,5 \\
0,5\end{array}$ & \begin{tabular}{r|}
22 \\
40,7 \\
3,9 \\
1,3 \\
\end{tabular} & \begin{tabular}{r|}
17 \\
31,5 \\
2,6 \\
1,0 \\
\end{tabular} & $\begin{array}{l}1 \\
1,9 \\
2,8 \\
0,1\end{array}$ & $\begin{array}{l}1 \\
1,9 \\
1,4 \\
0,1\end{array}$ & $\begin{array}{l}4 \\
7,4 \\
7,0 \\
0,2 \\
\end{array}$ & 54 & 3,3 & 0,74 & 39,7 & 2,6 \\
\hline $\begin{array}{l}\text { 15. "nichtlandw.» Er- } \\
\text { bengem. (mind. ein } \\
\text { Miterbe ist in } \\
\text { Grindelw. ansäss.) }\end{array}$ & $\begin{array}{r}8 \\
20,5 \\
3,1 \\
0,5 \\
\end{array}$ & $\begin{array}{r}12 \\
30,8 \\
2,2 \\
0,7 \\
\end{array}$ & $\begin{array}{r}15 \\
38,5 \\
2,3 \\
0,9 \\
\end{array}$ & $\begin{array}{l}0 \\
0 \\
0 \\
0\end{array}$ & $\begin{array}{l}0 \\
0 \\
0 \\
0\end{array}$ & $\begin{array}{r}4 \\
10,3 \\
7,0 \\
0,2 \\
\end{array}$ & 39 & 2,4 & 0,82 & 32,1 & 2,1 \\
\hline $\begin{array}{l}\text { 16. Einzeleigentümer } \\
\text { mit Wohnsitz in } \\
\text { der übrigen } \\
\text { Schweiz }\end{array}$ & \begin{tabular}{r|}
28 \\
16,1 \\
11,0 \\
1,6 \\
\end{tabular} & \begin{tabular}{|r|}
58 \\
33,3 \\
10,4 \\
3,3 \\
\end{tabular} & $\begin{array}{c}73 \\
42,0 \\
11,0 \\
4,2 \\
\end{array}$ & $\begin{array}{r}4 \\
2,3 \\
11,1 \\
0,2 \\
\end{array}$ & $\begin{array}{r}7 \\
4,0 \\
10,0 \\
0,4 \\
\end{array}$ & $\begin{array}{l}4 \\
2,3 \\
7,0 \\
0,2 \\
\end{array}$ & 174 & 10,6 & 0,70 & 123 & 8,2 \\
\hline $\begin{array}{l}\text { 17. Bund, Kanton, Ge- } \\
\text { meinden und Ver- } \\
\text { eine außerhalb } \\
\text { Grindelwalds }\end{array}$ & $\begin{array}{r}1 \\
14,3 \\
0,4 \\
0,1 \\
\end{array}$ & $\begin{array}{r}1 \\
14,3 \\
0,2 \\
0,1 \\
\end{array}$ & $\begin{array}{l}1 \\
14,3 \\
0,2 \\
0,1 \\
\end{array}$ & $\begin{array}{r}1 \\
14,3 \\
2,8 \\
0,1 \\
\end{array}$ & $\begin{array}{r}1 \\
14,3 \\
1,4 \\
0,1 \\
\end{array}$ & $\begin{array}{r}2 \\
28,6 \\
3,5 \\
0,1 \\
\end{array}$ & 7 & 0,4 & 0,38 & 2,7 & 0,2 \\
\hline $\begin{array}{l}\text { 18. auswärtige Erben- } \\
\text { gem. (alle Miterben } \\
\text { haben Wohnsitz } \\
\text { außerh. Grindelw.) }\end{array}$ & $\begin{array}{l}0 \\
0 \\
0 \\
0\end{array}$ & $\begin{array}{r}1 \\
33,3 \\
0,2 \\
0,1 \\
\end{array}$ & $\begin{array}{l}2 \\
66,7 \\
0,3 \\
0,1 \\
\end{array}$ & $\begin{array}{l}0 \\
0 \\
0 \\
0\end{array}$ & $\begin{array}{l}0 \\
0 \\
0 \\
0\end{array}$ & $\begin{array}{l}0 \\
0 \\
0 \\
0\end{array}$ & 3 & 0,2 & 0,78 & 2,4 & 0,2 \\
\hline $\begin{array}{l}\text { 19. Einzeleigentümer } \\
\text { mit Wohnsitz im } \\
\text { Ausland }\end{array}$ & $\begin{array}{r}4 \\
44,4 \\
1,6 \\
0,2 \\
\end{array}$ & $\begin{array}{r}3 \\
33,3 \\
0,5 \\
0,2 \\
\end{array}$ & $\begin{array}{l}2 \\
22,2 \\
0,3 \\
0,1 \\
\end{array}$ & $\begin{array}{l}0 \\
0 \\
0 \\
0 \\
\end{array}$ & $\begin{array}{l}0 \\
0 \\
0 \\
0 \\
\end{array}$ & $\begin{array}{l}0 \\
0 \\
0 \\
0\end{array}$ & 9 & 0,5 & 0,71 & 6,4 & 0,4 \\
\hline Total Parzellen & 254 & 558 & 663 & 36 & 70 & 57 & 1638 & 100,0 & 0,91 & 1503 & 100,0 \\
\hline$\%$ & 14,5 & 31,9 & 37,9 & 2,1 & 4,0 & 3,3 & & & & & \\
\hline & & & & & \multicolumn{2}{|c|}{$\begin{array}{l}\text { nicht berechen- } \\
\text { bare Fälle }\end{array}$} & 109 & 6,0 & 83 & 91,5 & 5,2 \\
\hline & & & & & \multicolumn{2}{|l|}{ Total } & \multicolumn{3}{|c|}{1747 Parzellen } & \multicolumn{2}{|c|}{1594,5 ha } \\
\hline
\end{tabular}

\section{Erläuterungen:}

1) 1. Zahl = Anzahl Parzellen, 2. Zahl = rel. Anteil an der Zeilensumme, 3. Zahl = rel. Anteil der Spaltensumme,

4. $Z$ ahl $=$ rel. Anteil an der Gesamtsumme

2) effektiv 12 Parzellen mit insgesamt rund 11 ha Fläche (vgl. Anm. 3)

3) Diese Fälle resultieren aus Inkongruenzen zwischen der Grundeigentumskartierung und der räumlichen MAB-GrindelwaldDatenbank.

Quellen: Eigene Erhebungen und Berechnungen auf der Basis der Flächenbeitragserhebung 1980, des amtlichen Grundbuches sowie von Feldbefragungen (vgl. Nägeli-Oertle 1986a, 100-101) 
überlegungen und völlig anderen Werten und Normen Schritt für Schritt aus dem angestammten Lebensraum gedrängt wird. ${ }^{7}$

Für Grindelwald mag diese Erfahrung des Überrolltwerdens allerdings nur für den "peripheren" Teil der landwirtschaftlichen Bevölkerung zutreffen, ist es doch einer beträchtlichen Anzahl Landwirte gelungen, sich ökonomisch am bisherigen Transformationsproze $\beta$ zu beteiligen.

Die Grindelwalder Bevölkerung und ihre Gemeindebehörden sind sich offensichtlich dieser Verdrängungsgefahr bewußt geworden, welche mit fortschreitender Entwicklung übrigens auch im Bereich des touristischen Angebotes selber manifest werden könnte! Sie haben zu diesem Zweck im vergangenen Jahr - unter anderem als Reaktion auf die vor «Überhitzungserscheinungen» warnenden MABUntersuchungen - ein "Leitbild Grindelwald 2000" erarbeitet (vgl. ARBEITSGRUPPE «MAB» DER GEMEINDE GRINDELWALD 1987), welches den Rahmen für eine aktive Steuerung der weiteren touristischen Entwicklung abgeben soll.

Dieses Leitbild setzt sich das anspruchsvolle Ziel, über das Jahr 2000 hinaus mittels einer ganzen Serie von Steuerungsempfehlungen die wirtschaftliche, kulturelle und nutzungsräumliche Entwicklung Grindelwalds derart zu beeinflussen, daß die ortsansässige Bevölkerung langfristig die Verfügungsgewalt über ihren eigenen Lebensraum beizubehalten vermag.

Im Rahmen dieses Leitbildes wird auch dem Ziel der Erhaltung einer funktionstüchtigen und traditionell ausgerichteten Landwirtschaft eine hohe Priorität eingeräumt. Bei der Verfolgung dieses Zieles wird es sich zeigen, daß nicht zuletzt der von uns untersuchten Grundeigentumsstruktur eine wichtige Rolle als Steuerfaktor der weiteren Entwicklung zukommt.

Eine zukünftige Wiederholung unserer Untersuchung wie auch ein geplantes langfristiges «Monitoring» der Gemeindeentwicklung werden zu zeigen haben, inwiefern es der Grindelwalder Bevölkerung gelingt, dieses Leitbild in die Wirklichkeit umzusetzen. Im Rahmen dieser Untersuchungen wird sich zudem auch die Gelegenheit bieten, der vorne angeführten These der sozialen Strukturierungsfunktion des Grundeigentums durch vertiefende Forschungen weiter nachzugehen.

\section{Anmerkungen}

1 Beim Forschungsprogramm MAB-Schweiz handelt es sich um ein von der UNESCO initiiertes und weitgehend vom SCHWEIZERISCHEN NATIONALFONDS finanziertes Nationales Forschungsprogramm zur Untersuchung der sozioökonomischen Entwicklung und ökologischen Belastung im schweizerischen Berggebiet. Die Gemeinde Grindelwald figurierte als eines von vier Testgebieten. Die definitiven Schlußberichte zum Programm Schweiz sowie zum Testgebiet Grindelwald befinden sich noch in der Vorbereitung. Die Schlußberichte zu den meisten Einzelprojekten sowie zu einzelnen Programm-Teilsynthesen sind unter der Federführung von Prof. P. Messerli in Bern erschienen und zu beziehen beim Bundesamt für Umweltschutz, Bibliothek, 3003 Bern. In unserem Zusammenhang sind v. a. die folgenden Berichte von Interesse: MEYRAT-SCHLEE (1983), PFISTER (1984), KUPFER/LANGENEGGER (1985), SCHEURER (1985), GROSJEAN u. a. (1986), WIESMANN (1986), MESSERLI u. a. (1986), LEIBUNDGUT (1987).

2 Ich danke meinen Kollegen A. Stalder für die Überlassung von einigem neueren statistischen Zahlenmaterial und Dr. U. Wiesmann für die freundliche Durchsicht des Manuskriptes.

${ }^{3}$ Die Bauzone ist auf der beigelegten Falzkarte ausgewiesen.

${ }^{4}$ Dieser Zusammenhang wird derzeit in der neuen angelsächsischen "Strukturationstheorie" weiter erforscht, in welcher es darum geht, das dialektische Zusammenwirken von räumlichen und institutionellen Strukturen einerseits und sozialen Handlungen andererseits besser verständlich zu machen (vgl. als Beispiel etwa PRED 1986).

5 Die kartographischen und erhebungstechnischen Probleme, welche bei dieser Kartierung zu lösen waren, sind ausführlich in NAEGELI-OERTLE (1986a: 253-257) beschrieben und brauchen deshalb hier nicht weiter erörtert zu werden.

6 Diese Unterscheidung ist nur auf dem detaillierteren Kartierungsoriginal möglich.

${ }^{7}$ Die Schmerzlichkeit dieser Erfahrung läßt sich vielleicht eher mit dichterischen Mitteln als mit trockenen wissenschaftlichen Zahlen vermitteln. Man lese hierzu etwa den Roman "Spätholz" von W. KAUER (1976), welcher sich allerdings auf die Situation eines Tessiner Bergtales bezieht.

8 Dieses Leitbild ist durch eine vom Grindelwalder Gemeinderat eingesetzte Arbeitsgruppe erarbeitet worden, welche vom MAB-GRINDELWALD-Projektkoordinator Dr. U. Wiesmann wissenschaftlich beraten worden ist. Nach einer außerordentlich breiten Vernehmlassung bei allen 79 Organisationen Grindelwalds (Behörden, Kommissionen, Bergschaften, Vereine) ist das Leitbild durch den Gemeinderat kürzlich offiziell in Kraft gesetzt worden.

\section{Literatur}

AEGERTER, R. (1983): Grindelwald. Beiträge zur Geschichte der Besiedlung und Landwirtschaft vom Mittelalter bis ins 19. Jahrhundert. Diss. am Geogr. Institut der Univ. Bern. Bern.

ARBEITSGRUPPE “MAB" DER GEMEINDE GRINDELWALD (1987): Leitbild Grindelwald 2000. Grindelwald.

BOEHM, H. (1980): Bodenmobilität und Bodenpreisgefüge in ihrer Bedeutung für die Siedlungsentwicklung. In: Bonner Geogr. Abhandlungen H. 65. Bonn.

GALLUSSER, W. A. (1979): Über die geographische Bedeutung des Grundeigentums. In: Geographica Helvetica 34 , 153-162. 
GROSJEAN, G. u. a. (1986): Ästhetische Bewertung ländlicher Räume. Am Beispiel von Grindelwald, im Vergleich mit anderen schweizerischen Räumen und in zeitlicher Veränderung. Schlußber. zum Schweiz. MAB-Programm Nr. 20 Bern.

KAUER, W. (1976): Spätholz. Zürich und Köln.

KUPFER, F. und LANGENEGGER, H. (1985): Der Wald in Grindelwald. Zustand, Produktion und Nutzungsmöglichkeiten. Schlußber. zum Schweiz. MAB-Programm Nr. 11. Bern.

LEIBUNDGUT, Ch. (1987): Hydroökologische Untersuchungen in einem alpinen Einzugsgebiet (Testgebiet Grindelwald). Schlußber. zum Schweiz. MAB-Programm Nr. 30. Berri.

LICHTENBERGER, E. (1979): Die Sukzession von der Agrarzur Freizeitgesellschaft in den Hochgebirgen Europas. In: Innsbrucker Geogr. Studien, Band 5, 401-436.

MESSERLI, P. u. a. (1986): Modellstudie Grindelwald. Zur Umweltverträglichkeit Olympischer Winterspiele im Berner Oberland 1996/2000. Schlußber. zum Schweiz. MAB-Programm Nr. 28. Bern.

MEYRAT-SCHLEE, E. (1983): Werte und Verhalten. Bedeutung und Wirkungsweise von Wertsystemen im Entwick- lungsprozeß einer Berggemeinde, aufgestellt am Beispiel von Grindelwald. Schlußber. Schweiz. MAB-Programm Nr. 2. Bern.

NAEGELI-OERTLE, R. (1986a): Die Berglandwirtschaft und Alpwirtschaft in Grindelwald. Schlußber. zum Schweiz. MAB-Programm Nr. 21. Bern.

NAEGELI-OERTLE, R. (1986b): Landwirtschaftliche Betriebsfallstudien im MAB-Testgebiet Grindelwald. Fachbeiträge zur Schweiz. MAB-Information Nr. 23. Bern.

PLANCK, U. und ZICHE, J. (1979): Land- und Agrarsoziologie. Stuttgart.

PRED, A. (1986): Place, Practice and Structure. Cambridge. SCHEURER, T. (1985): Bodenbildung und Bodenveränderung im Berggebiet (Testgebiet Grindelwald). Schlußber. zum Schweiz. MAB-Programm Nr. 12. Bern.

STALDER, A. (1988 i. E.): Zur Übertragbarkeit ausgewählter Problemstellungen der MAB-Pilotstudie Grindelwald auf andere Gemeinden des Berner Oberlandes. Diplomarbeit am Geogr. Institut der Univ. Bern. Bern.

WIESMANN, U. (1986): Wirtschaftliche, gesellschaftliche und räumliche Bedeutung des Fremdenverkehrs in Grindelwald. Schlußber. zum Schweiz. MAB-Programm Nr. 24. Bern. 